Johnny de Jesús Martinez martinez@peno.coppe.ufrj.br COPPE

Federal University of Rio de Janeiro - UFRJ Cidade Universitária 21945-970 Rio de Janeiro, RJ, Brazil

\author{
Paulo de Tarso T. Esperança \\ ptarso@peno.coppe.ufrj.br \\ COPPE \\ Federal University of Rio de Janeiro - UFRJ \\ Cidade Universitária \\ 21945-970 Rio de Janeiro, RJ, Brazil
}

\section{A Chebyshev Collocation Spectral Method for Numerical Simulation of Incompressible Flow Problems}

\begin{abstract}
This paper concerns the numerical simulation of internal recirculating flows encompassing a two-dimensional viscous incompressible flow generated inside a regularized square driven cavity and over a backward-facing step. For this purpose, the simulation is performed by using the projection method combined with a Chebyshev collocation spectral method. The incompressible Navier-Stokes equations are formulated in terms of the primitive variables, velocity and pressure. The time integration of the spectrally discretized, incompressible Navier-Stokes equations is performed by a secondorder mixed explicit/implicit time integration scheme. This scheme is a combination of the Crank-Nicolson scheme operating on the diffusive terms and a second-order AdamsBashforth scheme acting on the advective terms. The projection method is used to split the solution of the incompressible Navier-Stokes equations in two decoupled problems: the Burgers equation to predict an intermediate velocity field and the Poisson equation for the pressure, which is used to correct the intermediate velocity field and satisfy the continuity equation. Numerical simulations for flows inside a two-dimensional regularized square driven cavity for Reynolds numbers up to 10000 and over a backward-facing step for Reynolds numbers up to 875 are presented and compared with numerical results previously published, where good agreement is demonstrated.

Keywords: regularized square driven cavity, backward-facing step, chebyshev collocation spectral method, incompressible Navier-Stokes equations, projection method
\end{abstract}

\section{Introduction}

Internal recirculating flows generated within a bounded domain are very important under a technological perspective and also of a great scientific interest because they display several aspects of fluid mechanical phenomena in a very simple geometrical setting. Thus, corner vortices, longitudinal vortices, transition, and turbulence all occur naturally and can be studied in the same closed geometry (Shankar and Deshpande, 2000).

Numerical simulations of the Navier-Stokes equations for studying two-dimensional flows of incompressible viscous fluid are generally based upon a primitive variables formulation (velocity and pressure) or vorticity-streamfunction formulation. The major difficulty arising with the former formulation comes from the coupling of the pressure with the velocity, to satisfy the incompressibility condition. The continuity equation contains only velocity components, and there is no direct link with the pressure as it happens for compressible flow through the density (the lack of evolution equation for the pressure in primitive variables formulation is the source of difficulty). The use of a vorticitystreamfunction formulation of the equations avoids this problem. However, although its application to two-dimensional flows is quite common, the extension to three-dimensional problems is not straightforward. Thus, the primitive variables formulation is preferable because it is easily extended to three dimensions.

Several methods were proposed to overcome the difficulty arising in the primitive variables formulation. Among these, the projection methods or fractional steps methods (splitting methods) gained a new interest because of theirs non iterative nature, no requirement of any specific memory storage and appropriate use for simulation of unsteady incompressible flows. These methods belong to the predictor-corrector algorithms, where the pressure acts as a projection of the predicted velocity field (intermediate velocity field) onto a divergence-free space. In fact, there are several variants of the original projection method proposed by Chorin (1968) and Temam (1968). However, the use of projection methods has been

Paper accepted May, 2007. Technical Editor: Francisco R. Cunha. popularized associated to finite difference, volume or finite element methods, and there are few applications reported on spectral methods (Huges and Randriamampianina, 1998).

The main objective of this paper is to develop an efficient numerical method for the solution of a two dimensional incompressible viscous fluid with internal recirculating flows generated inside a bounded geometry. For this purpose, the numerical solution of incompressible Navier-Stokes equations in two dimensions (INSE2D) is based upon a Chebyshev collocation spectral method (also named Chebyshev pseudospectral method) in conjunction with a second-order projection method and coupled with appropriate boundary conditions. The motivation for using collocation spectral methods stems from their high precision and very low phase errors for the prediction of time-dependent flow regimes. A time integration of the equations system is performed by using a semi-implicit second-order accurate scheme (second-order Adams-Bashforth and Crank-Nicolson).

Spectral methods have been used in combination with temporal schemes of high order (at least order three). For example, Botella (1997) used a temporal scheme of order three, to improve the accuracy of his algorithm that involved a variant of the projection method with a pseudospectral method. In the present paper the algorithm is based on an original combination that involves the projection method with a semi-implicit temporal discretization of second order (which guarantees a good stability of the method) in a structure of spectral collocation. This numerical algorithm uses the technique of the complete diagonalization (non-iterative technique) which is very effective and fast for the direct solution of the resulting equations after the spatial and temporal discretization.

Two benchmark problems are chosen to assess the accuracy of the Chebyshev collocation spectral method. The first one deals with the regularized square driven cavity flow for Reynolds numbers up to 10000 . The second problem considers the flow over a backwardfacing step in a channel for Reynolds numbers up to 875 . Very good agreement is found between the numerical results of the present method and numerical results previously published by other authors.

The paper is organized as follows. At first, the mathematical formulation is presented, including the governing equations and the projection method. The next section is devoted to the numerical formulation, consisting of the temporal and spatial discretizations of 
the resulting equations, after the application of the projection method. In the following section the numerical results related to the two benchmark problems are presented and compared with numerical results previously published. The last section presents the conclusions.

\section{Nomenclature}

$c_{i}=$ coefficients to evaluate the first derivate matrix $D^{(1)}$

$D^{(1)}=$ Chebyshev collocation first derivative matrix

$D_{i k}^{(1)}=$ coefficients of matrix $D^{(1)}$

$H=1$, non-dimensional channel height

$H / 2$ = non-dimensional channel step height

$H_{\mathrm{s} 1}=$ horizontal extension at the bottom left of the secondary vortices

$H_{\mathrm{s} 2}=$ horizontal extension at the bottom right of the secondary vortices

$H_{\mathrm{s} 3}=$ horizontal extension at the top left of the secondary vortices

$h_{i}(x)=$ Lagrange polynomials

$L=30 H$, longitudinal non-dimensional channel length

$L_{c}=$ characteristic length (either length of the square cavity or channel height, $H$ )

$N=$ number of polynomials or collocation points in $x$

$M=$ number of polynomials or collocation points in $y$

$N_{b}=$ number of points indicating the starting of the Buffer zone

$N_{x}=$ number of points indicating the position of the outflow boundary

$P=\bar{P} / \rho U_{0}^{2}$, non-dimensional pressure field

$\bar{P}=$ dimensional pressure field

$\operatorname{Re}=U_{0} L_{c} / v$, Reynolds number characteristic of the flow

$s_{j}=$ general filter function

$t=\bar{t} U_{0} / L_{c}$, non-dimensional time

$\bar{t}=$ dimensional time

$u=$ non-dimensional horizontal component of velocity

$U_{0}=$ dimensional reference velocity

$u_{N}(x, t)=$ polynomial approximation of degree $N$ of the

function $u(x, t)$

$\hat{u}_{k}(t)=$ time dependent expansion spectral coefficients

$v=$ non-dimensional vertical component of velocity

$V=\bar{V} / U_{0}=(u, v)$, non-dimensional velocity vector

$\widetilde{V}=$ non-dimensional intermediate velocity vector at time $(n+1) \Delta t$

$\bar{V}=$ dimensional velocity vector

$\mathrm{V}_{\mathrm{s} 1}=$ vertical extension at the bottom left of the secondary vortices

$\mathrm{V}_{\mathrm{s} 2}=$ vertical extension at the bottom right of the secondary vortices

$V_{s 3}=$ vertical extension at the top left of the secondary vortices

$x=\bar{x} / L_{c}$, non-dimensional horizontal coordinate

$\bar{x}=$ dimensional horizontal coordinate

$x_{i}=$ Chebyshev-Gauss-Lobatto points

$\mathrm{x}_{\mathrm{c}}=$ horizontal position of the primary vortices

$\mathrm{x}_{\mathrm{s} 1}=$ horizontal position at the bottom left of the secondary vortices $\mathrm{x}_{\mathrm{s} 2}=$ horizontal position at the bottom right of the secondary vortices

$\mathrm{x}_{\mathrm{s} 3}=$ horizontal position at the top left of the secondary vortices

$y=\bar{y} / L_{c}$, non-dimensional vertical coordinate

$\bar{y}=$ dimensional vertical coordinate

$\mathrm{y}_{\mathrm{c}}=$ vertical position of the primary vortices

$\mathrm{y}_{\mathrm{s} 1}=$ vertical position at the bottom left of the secondary vortices

$\mathrm{y}_{\mathrm{s} 2}=$ vertical position at the bottom right of the secondary vortices

$\mathrm{y}_{\mathrm{s} 3}=$ vertical position at the top left of the secondary vortices

$w=$ boundary velocity

$w_{0}=$ initial velocity field

$T_{k}(x)=\cos \left(k \cos ^{-1} x\right)$, Chebyshev polynomials of order $k$

$T_{N}^{\prime}(x)=$ first-order derivative of the Chebyshev polynomial of order $N, T_{N}(x)$

\section{Greek Symbols}

$\Delta t=$ non-dimensional time step

$\Delta t_{c}=$ non-dimensional critical time step

$v=$ kinematic viscosity

$\omega=$ vorticity

$\rho=$ density

$\phi_{k}(x)=$ basis functions

$\psi=$ stream function

$\Omega=$ internal computational domain

$\partial \Omega=$ boundary of domain

$\wp=$ projection operator

$\delta_{i k}=$ Kronecker delta operator

$\hat{\tau}=$ unitary vector tangent to the boundary

$\hat{n}=$ unitary vector normal to the boundary

$\nabla=$ gradient operator

$\nabla^{2}=$ Laplacian operator

\section{Superscripts}

(1) (2) $^{(2)}$ order of the first and second derivative

${ }^{n},{ }^{n-1},{ }^{n+1}=$ variables evaluated at time $t, t-\Delta t, t+\Delta t$

' = indicates derivative with respect to $x$

\section{Subscripts}

$i, k=$ relative to the number of polynomials or collocation points

\section{Mathematical Formulation}

\section{Governing Equations}

Two-dimensional viscous incompressible fluid flows are governed by the Navier-Stokes equations. The dimensionless unsteady Navier-Stokes equations for incompressible flows in Cartesian coordinates may be written in primitive variables as

$$
\begin{aligned}
& \frac{\partial V}{\partial t}+(V . \nabla) V=-\nabla P+\frac{1}{\operatorname{Re}} \nabla^{2} V \quad \text { in } \quad \Omega \\
& \nabla . V=0 \quad \text { in } \quad \Omega
\end{aligned}
$$


where the unknowns are the vector $V$, which represents the velocity of the flow and the scalar $P$, which represents the pressure field. Here, $\operatorname{Re}$ is the Reynolds number of the flow, $U_{0}$ is the reference velocity, $L_{c}$ represents the characteristic length and $v$ the kinematical viscosity. Let $\Omega$ be the internal computational domain with sufficiently smooth boundaries $\partial \Omega$. The initial condition is given as

$$
\left.V\right|_{t=0}=w_{o} \quad \text { in } \quad \Omega
$$

satisfying Eq. (2) (the initial velocity field $w_{o}$ is solenoidal). Equations (1) and (2) are completed with an appropriate boundary condition for the velocity field,

$$
V=w \quad \text { on } \quad \partial \Omega
$$

The Navier-Stokes equations were non-dimensionalized using the following dimensionless variables:

$$
x=\frac{\bar{x}}{L_{c}}, y=\frac{\bar{y}}{L_{c}}, t=\frac{\bar{t} U_{0}}{L_{c}}, P=\frac{\bar{P}}{\rho U_{0}^{2}}, V=\frac{\bar{V}}{U_{0}}
$$

\section{Projection Method}

The major difficulty to solve numerically the incompressible Navier-Stokes equations (INSE) arises from the fact that the velocity $V$ and the pressure $P$ are coupled together by the incompressibility constraint (Eq. (2)). To overcome this difficulty, Chorin (1968) and Temam (1968), proposed the projection method (or fractional step method), which decouples the velocity and the pressure fields. The projection method has been widely used and has proven to be very efficient for this type of problem.

These classes of methods permit to uncouple the velocity and the pressure in each time step by reducing the solution of the Navier-Stokes equations to the solution of two successive problems. The first step solves an intermediate velocity, which does not satisfy the incompressibility condition (the velocity field is not solenoidal), while in the second step the intermediate velocity is projected onto a divergence-free space. This last step is equivalent to the solution of a Poisson equation for pressure, which is used to correct the intermediate velocity in order to fulfill the incompressibility condition.

The projection methods are based on the observation that the left-hand side of Eq. (1) is a Hodge decomposition. Hence an equivalent projection scheme is given by

$$
\frac{\partial V}{\partial t}=\wp\left[-(V . \nabla) V+\frac{1}{\operatorname{Re}} \nabla^{2} V\right]
$$

where $\wp$ is the operator which projects a vector field onto the space of divergence-free vector fields with appropriate boundary conditions.

The projection $\wp$ can be defined by the solution of a Poisson equation. Specifically, let $W=V+\nabla \phi$ be the Hodge decomposition of $W$, where $\phi$ is a scalar field and $V$ is the divergence-free velocity field that is required to satisfy $\left.V\right|_{\partial \Omega}=w$. Then, in order to determine $V$ from $W$ let (Brown, Cortez and Minion, 2001):

$$
V=\wp(W)=W-\nabla \phi
$$

$$
\begin{aligned}
& \nabla^{2} \phi=\nabla . W \quad \text { in } \quad \\
& \hat{n} \cdot \nabla \phi=\hat{n} .(W-w) \quad \text { on } \quad \partial \Omega
\end{aligned}
$$

Following Streett and Macaraeg (1989/90), the semi-discretized version of a semi-implicit projection method applied to Eqs. (1) and (2) can be written in two steps as follows:

a. The advection-diffusion step solves the intermediate velocity field at time $(n+1) \Delta t, \widetilde{V}$ by

$$
\frac{\widetilde{V}-V^{n}}{\Delta t}=\frac{1}{\operatorname{Re}} D(V)-N L(V) \quad \text { in } \quad \Omega
$$

with the intermediate boundary conditions

$$
\begin{aligned}
& \hat{\tau} \cdot \widetilde{V}=\hat{\tau} \cdot V+\hat{\tau} \cdot \Delta t\left(2 \nabla P^{n}-\nabla P^{n-1}\right) \quad \text { on } \quad \partial \Omega \\
& \hat{n} \cdot \widetilde{V}=\hat{n} \cdot V \quad \text { on } \quad \partial \Omega
\end{aligned}
$$

where $\quad N L(V)=(V . \nabla) V$ and $D(V)=\nabla^{2} V$ represent the advection and diffusion terms.

b. The pressure correction step solves the Poisson equation for $P^{n+1}$ from

$$
\nabla^{2} P^{n+1}=\frac{\nabla \cdot \widetilde{V}}{\Delta t} \quad \text { in } \quad \Omega
$$

with the homogeneous Neumann boundary condition:

$$
\hat{n} \cdot \nabla P^{n+1}=0 \quad \text { on } \quad \partial \Omega
$$

Then the velocities are updated with

$$
V^{n+1}=\widetilde{V}-\Delta t \nabla P^{n+1} \quad \text { in } \quad \Omega+\partial \Omega
$$

This step can be viewed as the projection of the velocity field onto the divergence-free space. At the end of the full step there exists a nonzero tangential component of the velocity on the boundary.

The magnitude of this finite slip velocity can be reduced by a proper choice of the intermediate boundary condition on $\widetilde{V}$ (Streett and Macaraeg, 1989/90). The slip velocity may be reduced to $O\left(\Delta t^{3}\right)$ through the boundary condition for the intermediate tangential velocity component, Eq. (11). This intermediate boundary condition is obtained from the Eq. (15) with an expansion of the term $\nabla P^{n+1}$ in Taylor series about $t=t^{n}$ and an approach of finite differences of the term $\nabla P_{t}^{n}$ (see Streett and Macaraeg, 1989/90).

\section{Numerical Formulation}

\section{Temporal Discretization}

The temporal integration used in the projection method is based on a second-order explicit-implicit scheme, which combines an explicit second-order Adams-Bashforth scheme for the advection terms and an implicit Crank-Nicolson scheme for dealing with the diffusion terms:

where 


$$
\begin{aligned}
& N L(V)=\frac{3}{2} N L\left(V^{n}\right)-\frac{1}{2} N L\left(V^{n-1}\right) \\
& D(V)=\frac{\left[D(\widetilde{V})+D\left(V^{n}\right)\right]}{2}
\end{aligned}
$$

Equations (16) and (17) are substituted in the Burgers equation (Eq. (10)), and at each time step, the solution of the problem reduces to the resolution of Burgers and Poisson equations.

\section{Spatial Discretization}

Equations obtained from the semi-discretized version of the projection method are spatially discretized using a Chebyshev collocation spectral method. The collocation spectral method is characterized by the fact that the numerical solution is forced to satisfy the governing equations exactly at collocation points. So, the series expansion for a function $u(x, t)$ on the domain $[-1,1]$ may be approximated as

$$
u_{N}(x, t)=\sum_{k=0}^{N} \hat{u}_{k}(t) \phi_{k}(x)
$$

where the $\phi_{k}(x)$ are the trial functions (also known as basis functions) and the $\hat{u}_{k}(t)$ are the time dependent expansion spectral coefficients.

For a Chebyshev collocation method, the functions $\phi_{k}(x)$ are the Chebyshev polynomials of order $k, T_{k}(x)$;

$$
u_{N}(x, t)=\sum_{k=0}^{N} \hat{u}_{k}(t) T_{k}(x)
$$

and the interpolation points are the so-called Chebyshev-GaussLobatto points,

$$
x_{i}=\cos \left(\frac{\pi i}{N}\right) \quad i=0,1, \ldots, N
$$

which correspond to the extreme points of the Chebyshev polynomials of order $N$,

$$
T_{N}(x)=\cos \left(N \cos ^{-1} x\right)
$$

The expansion coefficients, $\hat{u}_{k}(t)$ may be evaluated by the inverse relation

$$
\hat{u}_{k}(t)=\frac{2}{N c_{k}} \sum_{i=0}^{N} \frac{u_{N}\left(x_{i}, t\right)}{c_{i}} \cos \left(\frac{k \pi i}{N}\right), k=0,1, \ldots, N
$$

where $c_{i}$ and $c_{k}=1$ for $i, k=1,2, \ldots, N-1$ and $c_{0}=c_{N}=2$.

The collocation spectral method can be seen as a technique of interpolation, so Eq. (19) also can be expressed in terms of Lagrange polynomials de order $N, h_{i}(x)$ such that $h_{i}(x)=\delta_{i k}$, which is the Kronecker operator. The Lagrange polynomials for the ChebyshevGauss-Lobatto points (Eq. (20)) may be represented by the expression:

$$
h_{i}(x)=\frac{(-1)^{i+1}\left(1-x^{2}\right) T_{N}^{\prime}(x)}{\bar{c}_{i} N^{2}\left(x-x_{i}\right)}, \quad i=0,1, \ldots, N
$$

where $\bar{c}_{i}=1$ for $i=1, \ldots, N-1$ and $\bar{c}_{0}=\bar{c}_{N}=2$, (Canuto et al., 2006; Peyret, 2002).

The differentiation step can be accomplished in the transformed space ("transform method") or in physical space ("matrix multiplication method"). The first method is performed efficiently by means of a fast cosine transform with a recurrence relation in spectral space (see Canuto et al., 2006; Martinez, 1999) and the second method is based on explicit expressions obtained by differentiating the Lagrange polynomials. The matrix multiplication method is used in this paper because it is very efficient and easy to implement.

The spatial derivative of $u_{N}(x, t)$ at the collocation points $x_{i}$ is evaluated using the analytical derivative of the Lagrange polynomials,

$$
u_{N}^{\prime}\left(x_{i}, t\right)=\sum_{k=0}^{N} D_{i k}^{(1)} u\left(x_{k}, t\right), \quad i=0,1, \ldots, N
$$

where $D^{(1)}$ is the Chebyshev collocation derivative matrix. This matrix $D^{(1)}$ is given by the following expression:

$$
D_{i k}^{(1)}= \begin{cases}\frac{c_{i}(-1)^{i+k}}{c_{k}\left(x_{i}-x_{k}\right)}, & i \neq k, \\ -\frac{x_{k}}{2\left(1-x_{k}^{2}\right)}, & 1 \leq i=k \leq N-1, \\ \frac{2 N^{2}+1}{6}, & i=k=0, \\ -\frac{2 N^{2}+1}{6}, & i=k=N .\end{cases}
$$

This expression is easily found in the literature of the spectral methods (Boyd, 2001; Canuto et al., 2006; Deville et al., 2002; Peyret, 2002; Solomonoff and Turkel, 1989; Trefethen, 2000). The Chebyshev collocation second derivative matrix $D^{(2)}$ can be obtained analytically using an explicit expression (see Peyret, 2002) or by the following relation $D^{(2)}=\left(D^{(1)}\right)^{2}$.

The results presented so far can be readily extended to twodimensions. If an unknown matrix $U$ is defined such that $U\left(x_{i}, y_{j}\right)=u_{i j}$, then the partial derivatives of the interpolant evaluated at the collocation points, can be expressed in terms of matrix-matrix products, where differentiation with respect to $x$ corresponds to multiplying the rows of $D_{x}$ (collocation derivative matrix in $x$ ) by the columns of $U$, and differentiation with respect to $y$ corresponds to multiplying the rows of $U$ by the columns of $D_{y}^{T}$ (collocation derivative matrix transpose in $y$ ).

The algebraic system formed by the Helmholtz (Burgers) and Poisson equations obtained after the temporal and spatial discretizations has to be solved at each time step, using a complete diagonalization of the operators in both directions. This matrix diagonalization scheme was introduced by Lynch et al. (1964) for Finite Difference Methods and considered for Spectral Methods by Ehrenstein and Peyret (1989), Zhao and Yedlin (1994) and especially for the solution of the Poisson equation in polar and cylindrical coordinates by Chen et al. (2000). The computation of eigenvalues, eigenvectors and the inversion of the corresponding matrices are performed once in a preprocessing step before starting the time integration. Thus, at each time step, the solution is obtained 
from simple product of matrices (see Peyret, 2002; Chen et al., 2000; Martinez, 2005).

\section{Numerical Results}

\section{Regularized Square Driven Cavity Flow}

In order to take advantage of the high accuracy of the Chebyshev collocation spectral method, a regularized square driven cavity is considered, where the singularity at the upper corners is removed using a parabolic horizontal velocity distribution instead of a constant velocity equal to one (the usual driven cavity flow).

The regularized square driven cavity (see Fig. 1a) is a model for flow in a cavity where the upper boundary moves to the right with a horizontal velocity distribution of $16 x^{2}(1-x)^{2}$, while the other three boundaries are kept stationary (no-slip boundary conditions) and this generates the internal recirculating flow in the cavity. The horizontal velocity distribution produces a maximum velocity of $u_{\max }=1.0$ and the Reynolds number in this problem is defined by the following relation:

$$
\operatorname{Re}=u_{\max } L_{c} / v=1.0 / v
$$

The initial condition for the cases of $\operatorname{Re} \leq 1000$ starts from rest. The initial condition for the cases of $\operatorname{Re} \geq 2000$ is the steady state solution of the previous Reynolds number in order to accelerate the temporal integration process.

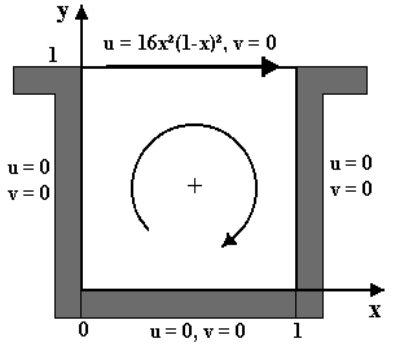

(a)

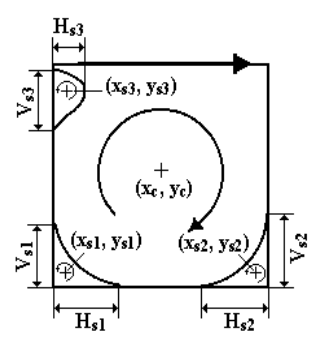

(b)
Figure 1. Regularized square driven cavity flow: (a) Boundary conditions; (b) Flow configuration and nomenclature (Tanahashi and Okanaga, 1990).

The time accuracy of the method is checked on the steady-state solution of the regularized square driven cavity flow for three values of the Reynolds number, $\mathrm{Re}=100,400$ and 1000 . To measure the temporal accuracy, the discrete norm of the divergence on the inner collocation points, $\|\nabla . V\|$ has been considered:

$$
\|\nabla \cdot V\|^{2}=\frac{1}{(N-1)(M-1)} \sum_{i=2}^{N-1} \sum_{j=2}^{M-1}\left[\nabla \cdot V\left(x_{i}, y_{j}\right)\right]^{2}
$$

Figure 2 shows the variation of the discrete norm of the divergence on the inner collocation points with the time step $\Delta t$ for the steady-state solutions of three values at $R e=100,400$ and 1000 on a grid of Chebyshev of $33 \times 33$. In this figure, it can be noticed that as the time step diminishes the discrete norm of the divergence also diminishes (order of accuracy increases). Our numerical experiments indicate that the critical time step $\Delta t_{c}$ (maximal time step to guarantee the stability of the method) varies with the Reynolds number. Thus, for a Reynolds number of 100 it is necessary a $\Delta t \leq 0.04\left(\Delta t_{c}=0.04\right)$ for stability of the scheme, while that, for a Reynolds number of 1000 a value of
$\Delta t \leq 0.01\left(\Delta t_{c}=0.01\right)$ is required for the scheme to become stable. Then, the choice of a time step of 0.001 is enough to guarantee the stability of the method as well as a high precision for all the Reynolds numbers to be studied.

To study the convergence of the solution with respect to the spatial resolution, the stream function $\psi$ and the vorticity $\omega$ associated with the regularized cavity flow has been considered. Thus, at each time step, the vorticity $\omega$ is computed according to

$$
\omega^{n+1}=\frac{\partial v^{n+1}}{\partial x}-\frac{\partial u^{n+1}}{\partial y}
$$

and the steady-state solution is reached when

$$
\frac{\sum_{i=2}^{N-1} \sum_{j=2}^{M-1} \max \left|\omega_{i, j}^{n+1}-\omega_{i, j}^{n}\right|}{\Delta t \sum_{i=2}^{N-1} \sum_{j=2}^{M-1} \max \left|\omega_{i, j}^{n+1}\right|}<2 \times 10^{-8}, \quad n=0,1,2, \ldots
$$

The streamfunction $\psi$ is computed using the following Poisson equation

$$
\begin{aligned}
& \nabla^{2} \psi^{n+1}=-\omega^{n+1} \quad \text { in } \quad \Omega \\
& \psi^{n+1}=0 \quad \text { on } \quad \partial \Omega
\end{aligned}
$$

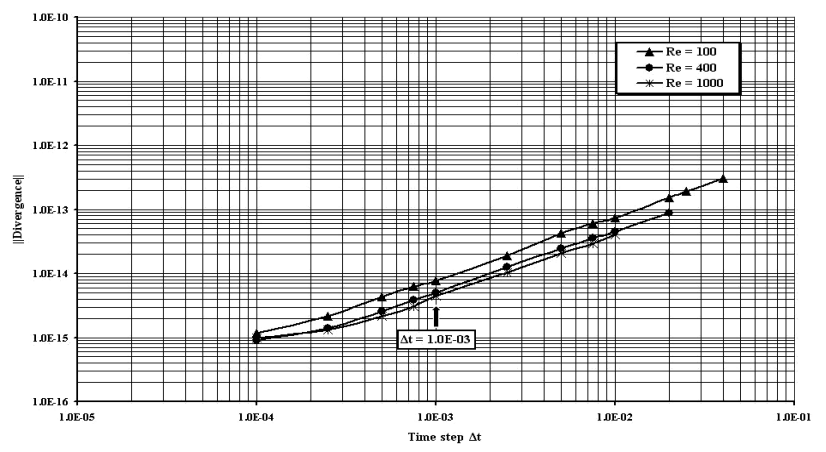

Figure 2. Variation of the discrete norm of the divergence with the time step $\Delta t$ on a grid of Chebyshev of $33 \times 33$.

The convergence is assessed by considering the steady-state regularized driven cavity flow for two values of $\mathrm{Re}=100$ and 400 . The comparisons of some characteristic flow variables are made with those of Botella (1997), who used a third-order time accurate Chebyshev projection scheme for approximating the Navier-Stokes incompressible equations, and Ehrenstein and Peyret (1989), who used a Chebyshev collocation method for solving the Navier-Stokes equations based upon a vorticity-stream function formulation. The comparisons were carried out for different spatial resolutions on the maximal value of the streamfunction $|\psi|$ on the inner collocation points and the maximal value of the vorticity $|\omega|$ on the moving top side $(x, 1)$ :

$$
\begin{aligned}
& M_{1}=\max \left|\psi\left(x_{i}, y_{j}\right)\right| \text { on the inner collocation points; } \\
& M_{2}=\max \left|\omega\left(x_{i}, 1\right)\right| \text { on the collocation points of the upper side } \\
& =1 . \\
& M_{3}=\max |\omega(x, 1)| \quad \text { from solution interpolated on } 201 \text { equally }
\end{aligned}
$$$$
y=1 \text {. }
$$
spaced points on $y=1$. 
Tables 1 and 2, respectively, display these comparisons. For $\mathrm{Re}=100$, the results of the present method for $M_{1}$ and $M_{2}$ are in good agreement with the results obtained by Botella (1997) and also by Ehrenstein and Peyret (1989) for a grid $N=M>17$. For $\operatorname{Re}=$ 400, the values of $M_{1}$ and $M_{2}$ obtained by the present method agree well with those results obtained by Botella (1997) and by Ehrenstein and Peyret (1989) for a grid $N=M>21$. The evolution of $M_{1}$ and $M_{2}$, when the spatial resolution $N$ increases, is not the best indication of convergence of the solution. The sensitive quantity such as $M_{2}$ does not give a precise account of the maximal value of the vorticity $\omega$, because of the strong variation of the vorticity on the moving top side $\omega(x, 1)$, and the unequal distribution of the Chebyshev-Gauss-Lobatto points. So, it is much more significant to consider the maximal value of the polynomial $\omega_{N}(x, 1)$ on $x \in[-1,1]$. From the knowledge of the grid values $\omega\left(x_{i}, 1\right), i=1,2, \ldots, N$, the continuous polynomial $\omega_{N}(x, 1)$ is reconstructed through the Chebyshev expansion (Eq. 19) after having calculated the Chebyshev coefficient with Eq. (22). Thus, the maximal value of the polynomial $\omega_{N}(x, 1)$ taken on 201 equally spaced points on the moving top side is denoted by $M_{3}$ (Peyret, 2002).

The evolution of the quantity $M_{3}$, when $N$ increases, provides a monotonic convergence of the numerical solution. For a sufficient number of collocation points, the present method converges for very near values to those obtained by Botella (1997) and Ehrenstein and Peyret (1989) (see Table 1 and 2). Thus, for $\mathrm{Re}=100$ and 400 , the values de $M_{3}$ obtained by the present method for $N=33 \quad\left(M_{3}=13.4443\right.$ and $\left.M_{3}=24.9110\right)$ and $N=41 \quad\left(M_{3}=13.4444\right.$ and $\left.M_{3}=24.9109\right)$ show that 33 collocation points are sufficient to represent correctly a regularized

Table 1. Comparison of the characteristic flow variables $M_{1}, M_{2}, M_{3}$ for the regularized square driven cavity at $R e=100$.

\begin{tabular}{|c|c|c|c|c|c|c|c|c|}
\hline$N=M$ & \begin{tabular}{c}
\multicolumn{1}{c}{$M_{1}$} \\
Present \\
Method
\end{tabular} & $\begin{array}{c}M_{1} \\
\text { Botella (1997) }\end{array}$ & $\begin{array}{c}\quad M_{2} \\
\text { Present } \\
\text { Method }\end{array}$ & $\begin{array}{c}M_{2} \\
\text { Botella (1997) }\end{array}$ & $\begin{array}{l}\quad M_{2} \\
\text { Ehrenstein \& } \\
\text { Peyret (1989) }\end{array}$ & $\begin{array}{c}\quad M_{3} \\
\text { Present } \\
\text { Method }\end{array}$ & $\begin{array}{c}M_{3} \\
\text { Botella (1997) }\end{array}$ & $\begin{array}{l}\quad M_{3} \\
\text { Ehrenstein \& } \\
\text { Peyret (1989) }\end{array}$ \\
\hline 17 & $8.3115 \mathrm{E}-02$ & $8.3160 \mathrm{E}-02$ & 13.3347 & 13.3467 & 13.3687 & 13.4628 & 13.4476 & 13.4663 \\
\hline 21 & 8.2673E-02 & 8.2694E-02 & 13.1869 & 13.1759 & 13.1780 & 13.4472 & 13.4441 & 13.4459 \\
\hline 25 & 8.3306E-02 & $8.3315 \mathrm{E}-02$ & 13.4291 & 13.4226 & 13.4227 & 13.4449 & 13.4446 & 13.4446 \\
\hline 33 & $8.3400 \mathrm{E}-02$ & 8.3402E-02 & 13.3441 & 13.3423 & 13.3422 & 13.4443 & 13.4448 & 13.4447 \\
\hline 41 & 8.3595E-02 & - & 13.4430 & - & - & 13.4444 & 13.4447 & - \\
\hline
\end{tabular}

Table 2. Comparison of the characteristic flow variables $M_{1}, M_{2}, M_{3}$ for the regularized square driven cavity at $R e=400$.

\begin{tabular}{|c|c|c|c|c|c|c|c|c|}
\hline$N=M$ & $\begin{array}{c}\quad M_{1} \\
\text { Present } \\
\text { Method }\end{array}$ & $\begin{array}{c}M_{1} \\
\text { Botella (1997) }\end{array}$ & $\begin{array}{c}\quad M_{2} \\
\text { Present } \\
\text { Method }\end{array}$ & $\begin{array}{c}M_{2} \\
\text { Botella (1997) }\end{array}$ & $\begin{array}{c}\quad M_{2} \\
\text { Ehrenstein \& } \\
\text { Peyret (1989) }\end{array}$ & $\begin{array}{c}\quad M_{3} \\
\text { Present } \\
\text { Method }\end{array}$ & $\begin{array}{c}M_{3} \\
\text { Botella (1997) }\end{array}$ & $\begin{array}{c}\quad M_{3} \\
\text { Ehrenstein \& } \\
\text { Peyret (1989) }\end{array}$ \\
\hline 17 & $8.5341 \mathrm{E}-02$ & $8.5777 \mathrm{E}-02$ & 24.7189 & 24.7799 & 25.2329 & 25.0855 & 25.1604 & 25.4675 \\
\hline 21 & $8.5113 \mathrm{E}-02$ & $8.5192 \mathrm{E}-02$ & 24.6189 & 24.6268 & 24.6693 & 24.9362 & 24.9273 & 24.9846 \\
\hline 25 & $8.5671 \mathrm{E}-02$ & $8.5716 \mathrm{E}-02$ & 24.9172 & 24.9157 & 24.9344 & 24.9176 & 24.9148 & 24.9333 \\
\hline 33 & $8.5467 \mathrm{E}-02$ & 8.5480E-02 & 24.7821 & 24.7845 & 24.7845 & 24.9110 & 24.9111 & 24.9110 \\
\hline 41 & 8.5893E-02 & - & 24.8622 & - & - & 24.9108 & 24.9109 & - \\
\hline
\end{tabular}

cavity flow, the relative difference between $M_{3}$ obtained for $N=33$ and for $N=41$ is less than $8 \mathrm{E}-06$. This relative difference is the same for the values de $M_{3}$ obtained by Botella (1997).

Then, to represent well the steady-state flow with the vortices inside of the regularized cavity for all the numbers of Reynolds a mesh of $33 \times 33$ was used in combination with a time step fixed of 0.001 to guarantee a high precision and stability of the present method as well as avoiding high computational cost.

The flow configuration is characterized by the magnitude and the location of the centers of the primary and secondary vortices. The steady state streamfunction contours were computed solving the equation of Poisson (Eqs. (30) - (31)). The center of the primary vortex was defined using the criterion of the location of the minimum value of the streamfunction. The centers of the secondary vortices were defined using the criterion of the location of the maximum value of the streamfunction and the centers of the vortices tertiary were defined using the criterion of the location of the minimum value of the streamfunction.

Table 3 shows the comparison of the some characteristic values of the regularized square driven cavity flow with previously published numerical results obtained by Shen (1991), according to the nomenclature in Fig. 1b. Shen (1991) used the projection scheme of Kim and Moin (1985) in conjunction with a ChebyshevTau space discretization. Shen (1991) results were based on uniform grids of $17 \times 17$ up to $49 \times 49$ depending on the Reynolds numbers (see Table 3). Although we have used only a $33 \times 33$ grid for all cases, the solutions are in good agreement.

The numerical results obtained by Ghia et al. (1982) for the driven cavity flow (not-regularized) for Reynolds numbers up to 10000 are also shown in the Table 3. Ghia et al. (1982) used the vorticity-streamfunction formulation of incompressible NavierStokes equations and a multigrid method, with a 129x129 uniform mesh at $\operatorname{Re}=1000$ and a $257 \times 257$ uniform mesh at $\mathrm{Re} \geq 5000$.

The comparison of the some characteristic values of the regularized driven cavity flow with numerical results obtained by Ghia et al. (1982) shows that the vortex dynamics between the two cavity flows (regularized and not-regularized) is very similar although the quantitative characteristics are somewhat different.

Figures $3 \mathrm{a}$ - 3f show the steady state streamlines of the regularized square driven cavity flow for Reynolds numbers up to 10000. In these figures can be noted the variation of the magnitude 
and location of the centers of the primary and secondary vortices with Reynolds numbers. The some streamfunction contour values for all Reynolds number are shown in theses Figures.

In the Figs. $3 \mathrm{a}, 3 \mathrm{~b}$ and $3 \mathrm{c}$ can also be observed the formation and growing of the secondary vortices at the bottom left and bottom right of the cavity when the Reynolds number increases. These figures show mainly that the secondary vortices for the Reynolds numbers studied can be very well represented using only $33 \times 33$ points of collocation, thanks to the condensed distribution of the Chebyshev-Gauss-Lobatto points near the boundary.
Figures $3 \mathrm{~d}, 3 \mathrm{e}$ and $3 \mathrm{f}$ show the streamlines of the steady flow for three Reynolds numbers $(\operatorname{Re}=2000,5000$ and 10000). Once again, can be observed the formation, evolution and growing of another secondary vortex that appears at the top left of the regularized cavity. At $\mathrm{Re}=10000$, a tertiary vortex becomes visible at the bottom right of the cavity with center in $(0.945,0.04)$ and another small tertiary vortex begins to appear at the top right corner (see Fig. 3f). These figures show than the secondary and tertiary vortices for Reynolds numbers up 10000 can be very well represented with only $33 \times 33$ points of Chebyshev collocation.

Table 3. Comparison of some characteristic values of the regularized square driven cavity flow.

\begin{tabular}{|c|c|c|c|c|c|}
\hline $\operatorname{Re}$ & Parameter & $\begin{array}{c}\text { Present Method } \\
(*) \\
\text { (Regularized) }\end{array}$ & $\begin{array}{c}\text { Shen (1991) } \\
\text { (Regularized) }\end{array}$ & (Grid) & $\begin{array}{r}\text { Ghia et al. (1982) } \\
\text { (Not-Regularized) }\end{array}$ \\
\hline \multirow{5}{*}{100} & $\left(\mathrm{x}_{\mathrm{c}}, \mathrm{y}_{\mathrm{c}}\right)$ & $(0.607,0.753)$ & $(0.609,0.750)$ & \multirow{5}{*}{$(17 \times 17)$} & $(0.6172,0.7344)$ \\
\hline & $\left(\mathrm{x}_{\mathrm{s} 1}, \mathrm{y}_{\mathrm{s} 1}\right)$ & $(0.032,0.032)$ & $(0.031,0.031)$ & & - \\
\hline & $\left(\mathrm{x}_{\mathrm{s} 2}, \mathrm{y}_{\mathrm{s} 2}\right)$ & $(0.955,0.052)$ & $(0.953,0.047)$ & & - \\
\hline & $\left(\mathrm{H}_{\mathrm{s} 1}, \mathrm{~V}_{\mathrm{s} 1}\right)$ & $(0.13,0.14)$ & - & & - \\
\hline & $\left(\mathrm{H}_{\mathrm{s} 2}, \mathrm{~V}_{\mathrm{s} 2}\right)$ & $(0.14,0.14)$ & - & & - \\
\hline \multirow{5}{*}{400} & $\left(x_{c}, y_{c}\right)$ & $(0.578,0.615)$ & $(0.578,0.625)$ & \multirow{5}{*}{$(17 \times 17)$} & $(0.5547,0.6055)$ \\
\hline & $\left(\mathrm{x}_{\mathrm{s} 1}, \mathrm{y}_{\mathrm{s} 1}\right)$ & $(0.045,0.041)$ & $(0.031,0.047)$ & & \\
\hline & $\left(\mathrm{x}_{\mathrm{s} 2}, \mathrm{y}_{\mathrm{s} 2}\right)$ & $(0.900,0.115)$ & $(0.922,0.094)$ & & $(0.8906,0.1250)$ \\
\hline & $\left(\mathrm{H}_{\mathrm{s} 1}, \mathrm{~V}_{\mathrm{s} 1}\right)$ & $(0.135,0.110)$ & - & & \\
\hline & $\left(\mathrm{H}_{\mathrm{s} 2}, \mathrm{~V}_{\mathrm{s} 2}\right)$ & $(0.250,0.315)$ & - & & $(0.2167,0.3203)$ \\
\hline \multirow{5}{*}{1000} & $\left(\mathrm{x}_{\mathrm{c}}, \mathrm{y}_{\mathrm{c}}\right)$ & $(0.545,0.575)$ & $(0.547,0.578)$ & \multirow{5}{*}{$(25 \times 25)$} & $(0.5313,0.5625)$ \\
\hline & $\left(\mathrm{x}_{\mathrm{s} 1}, \mathrm{y}_{\mathrm{s} 1}\right)$ & $(0.077,0.068)$ & $(0.078,0.063)$ & & $(0.0859,0.0781)$ \\
\hline & $\left(\mathrm{x}_{\mathrm{s} 2}, \mathrm{y}_{\mathrm{s} 2}\right)$ & $(0.876,0.118)$ & $(0.922,0.094)$ & & $(0.8594,0.1094)$ \\
\hline & $\left(\mathrm{H}_{\mathrm{s} 1}, \mathrm{~V}_{\mathrm{s} 1}\right)$ & $(0.205,0.170)$ & - & & $(0.2188,0.1680)$ \\
\hline & $\left(\mathrm{H}_{\mathrm{s} 2}, \mathrm{~V}_{\mathrm{s} 2}\right)$ & $(0.320,0.335)$ & - & & $(0.3034,0.3536)$ \\
\hline \multirow{7}{*}{2000} & $\left(x_{c}, y_{c}\right)$ & $(0.535,0.555)$ & $(0.531,0.547)$ & \multirow{7}{*}{$(33 \times 33)$} & - \\
\hline & $\left(\mathrm{x}_{\mathrm{s} 1}, \mathrm{y}_{\mathrm{s} 1}\right)$ & $(0.09,0.09)$ & $(0.094,0.094)$ & & - \\
\hline & $\left(\mathrm{x}_{\mathrm{s} 2}, \mathrm{y}_{\mathrm{s} 2}\right)$ & $(0.856,0.107)$ & $(0.922,0.094)$ & & - \\
\hline & $\left(\mathrm{x}_{\mathrm{s} 3}, \mathrm{y}_{\mathrm{s} 3}\right)$ & $(0.028,0.888)$ & $(0.031,0.908)$ & & - \\
\hline & $\left(\mathrm{H}_{\mathrm{s} 1}, \mathrm{~V}_{\mathrm{s} 1}\right)$ & $(0.255,0.195)$ & - & & - \\
\hline & $\left(\mathrm{H}_{\mathrm{s} 2}, \mathrm{~V}_{\mathrm{s} 2}\right)$ & $(0.35,0.35)$ & - & & - \\
\hline & $\left(\mathrm{H}_{\mathrm{s} 3}, \mathrm{~V}_{\mathrm{s} 3}\right)$ & $(0.05,0.14)$ & - & & - \\
\hline \multirow{7}{*}{5000} & $\left(\mathrm{x}_{\mathrm{c}}, \mathrm{y}_{\mathrm{c}}\right)$ & $(0.518,0.543)$ & $(0.516,0.531)$ & \multirow{7}{*}{$(33 \times 33)$} & $(0.5117,0.5352)$ \\
\hline & $\left(\mathrm{x}_{\mathrm{s} 1}, \mathrm{y}_{\mathrm{s} 1}\right)$ & $(0.081,0.121)$ & $(0.094,0.094)$ & & $\begin{array}{c}(0.0703,0.1367) \\
(0.8086,0.0742)\end{array}$ \\
\hline & $\left(\mathrm{x}_{\mathrm{s} 2}, \mathrm{y}_{\mathrm{s} 2}\right)$ & $(0.818,0.081)$ & $(0.922,0.094)$ & & $(0.0625,0.9102)$ \\
\hline & $\left(\mathrm{x}_{\mathrm{s} 3}, \mathrm{y}_{\mathrm{s} 3}\right)$ & $(0.082,0910)$ & $(0.078,0.908)$ & & $(0.3184,0.2643)$ \\
\hline & $\left(\mathrm{H}_{\mathrm{s} 1}, \mathrm{~V}_{\mathrm{s} 1}\right)$ & $(0.350,0.270)$ & - & & $\begin{array}{l}(0.3565,0.4180) \\
(0.1211,0.2693)\end{array}$ \\
\hline & $\left(\mathrm{H}_{\mathrm{s} 2}, \mathrm{~V}_{\mathrm{s} 2}\right)$ & $(0.370,0.415)$ & - & & \\
\hline & $\left(\mathrm{H}_{\mathrm{s} 3}, \mathrm{~V}_{\mathrm{s} 3}\right)$ & $(0.130,0.260)$ & - & & \\
\hline \multirow{7}{*}{10000} & $\left(x_{c}, y_{c}\right)$ & $(0.509,0.523)$ & $(0.516,0.531)$ & \multirow{7}{*}{$(49 x 49)$} & $(0.5117,0.5333)$ \\
\hline & $\left(\mathrm{x}_{\mathrm{s} 1}, \mathrm{y}_{\mathrm{s} 1}\right)$ & $(0.080,0.140)$ & $(0.094,0.094)$ & & $(0.0586,0.1641)$ \\
\hline & $\left(\mathrm{x}_{\mathrm{s} 2}, \mathrm{y}_{\mathrm{s} 2}\right)$ & $(0.772,0.063)$ & $(0.922,0.094)$ & & $\begin{array}{l}(0.7656,0.0586) \\
(0.0703,0.9141)\end{array}$ \\
\hline & $\left(\mathrm{x}_{\mathrm{s} 3}, \mathrm{y}_{\mathrm{s} 3}\right)$ & $(0.090,0.915)$ & $(0.094,0.908)$ & & $(0.3438,0.2891)$ \\
\hline & $\left(\mathrm{H}_{\mathrm{s} 1}, \mathrm{~V}_{\mathrm{s} 1}\right)$ & $(0.350,0.300)$ & - & & $(0.3906,0.4492)$ \\
\hline & $\left(\mathrm{H}_{\mathrm{s} 2}, \mathrm{~V}_{\mathrm{s} 2}\right)$ & $(0.390,0.440)$ & - & & $(0.1589,0.3203)$ \\
\hline & $\left(\mathrm{H}_{\mathrm{s} 3}, \mathrm{~V}_{\mathrm{s} 3}\right)$ & $(0.173,0.315)$ & - & & \\
\hline
\end{tabular}

(*) - All results using a $33 \times 33$ grid

Shen (1991) found a stationary solution up to $\mathrm{Re}=10000$. He found that the first Hopf bifurcation (when the steady flow loses its stability to the benefit of a periodic flow) took place at a critical value located between $10000<\operatorname{Re}<10500$. At $\operatorname{Re}=16000$, the 
computed solution loses the time periodicity and becomes quasiperiodic, which indicated that another bifurcation occurs at a critical Reynolds number between $15000<\operatorname{Re}<16000$. Botella (1997) used a third-order time accurate Chebyshev projection scheme to compute the flow at $\operatorname{Re}=10300$, starting from steady solution at $\operatorname{Re}=10000$ and the flow reached a periodic state.

In our study, we found that the flow converges to steady state for Reynolds numbers up to 10000 and our numerical results did not show any evidence of a Hopf bifurcation, in agreement with the

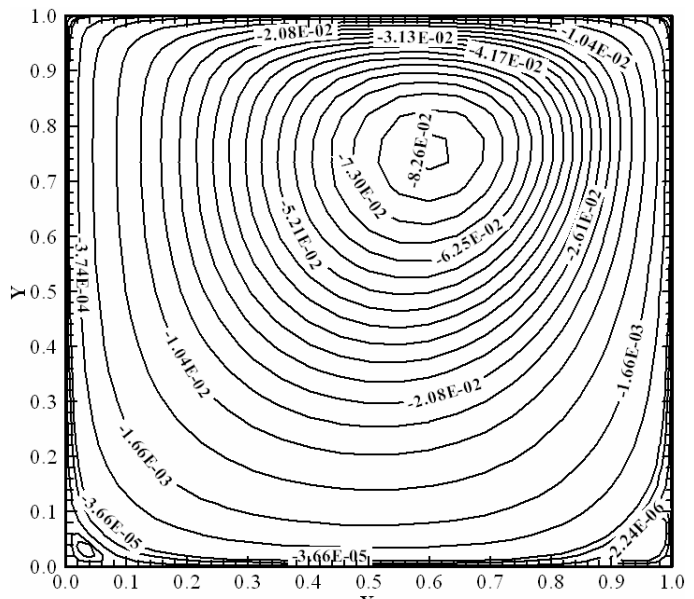

(a) ${ }^{\mathrm{X}}$

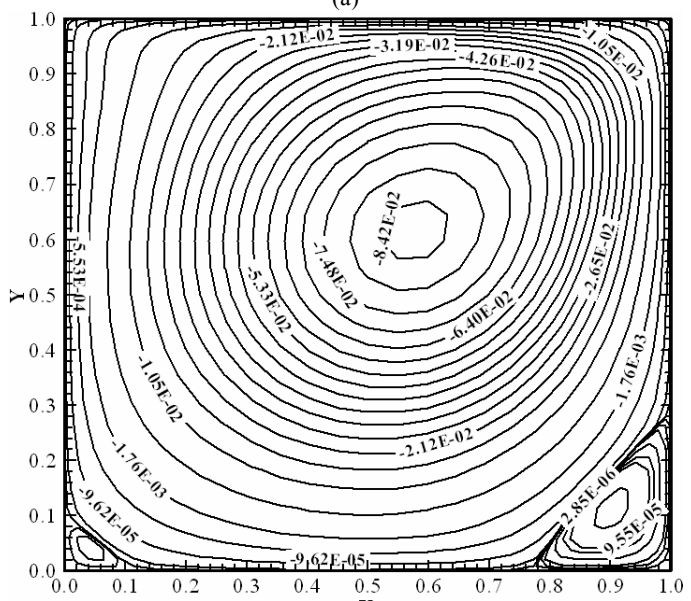

(b)

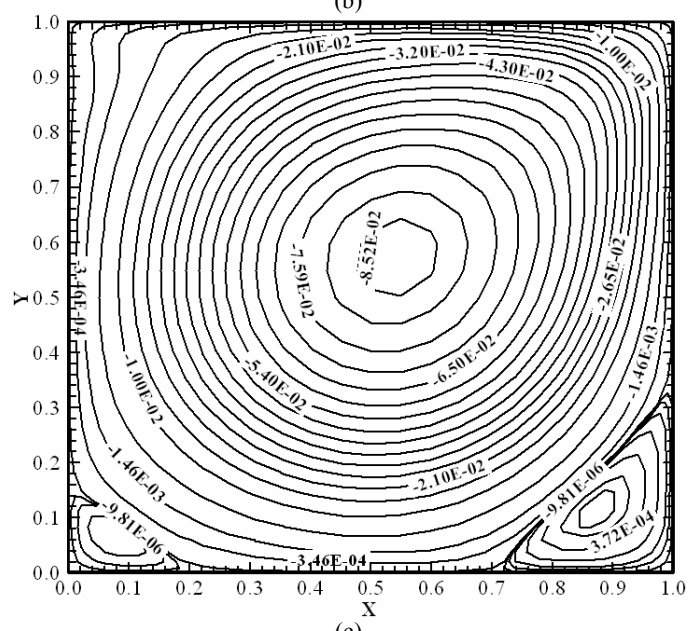

(c)

Figure 3. Steady state streamlines of the regularized square driven cavity flow: (a) $\operatorname{Re}=100$, (b) $\operatorname{Re}=400$, (c) $\operatorname{Re}=1000$, (d) $\operatorname{Re}=2000$, (e) $\operatorname{Re}=5000$, (f) $\operatorname{Re}=10000$.

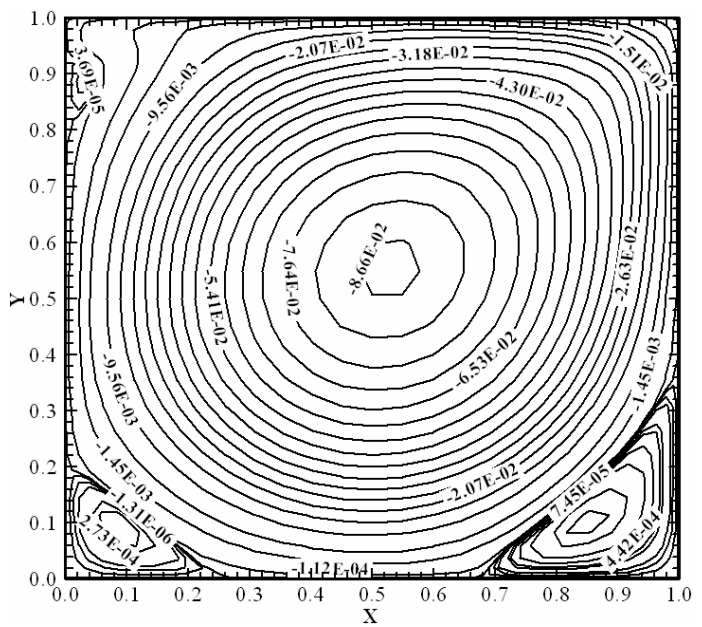

(d)

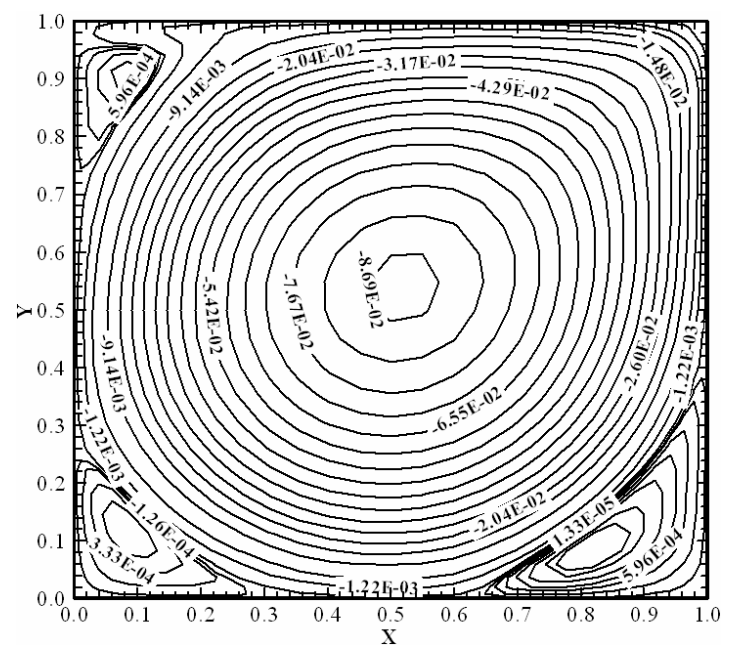

(e)

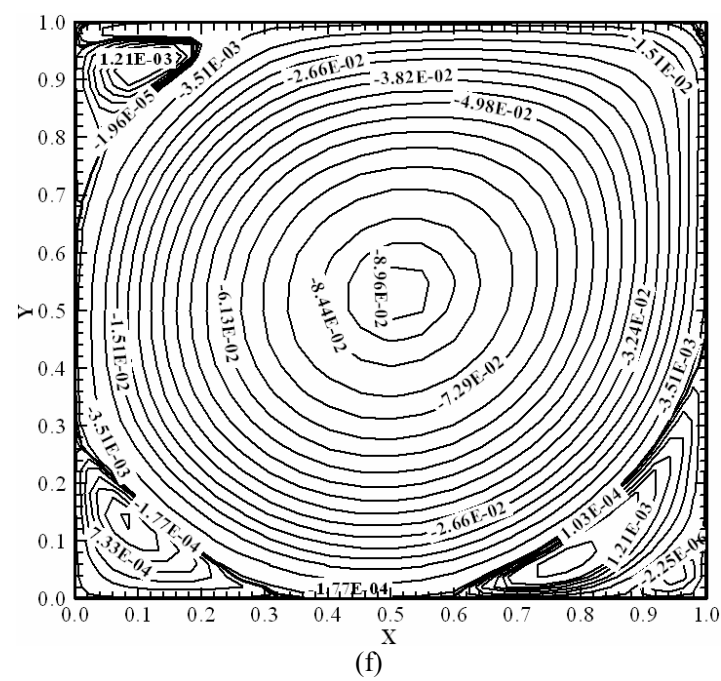

Figure 3. (Continued).

results obtained by Shen (1991) and Botella (1997).

Finally, the $u$ and $v$ velocity component profiles along the centerlines of the regularized square driven cavity for Reynolds numbers up to 10000 are shown in the Fig. 4a and Fig.4b. 


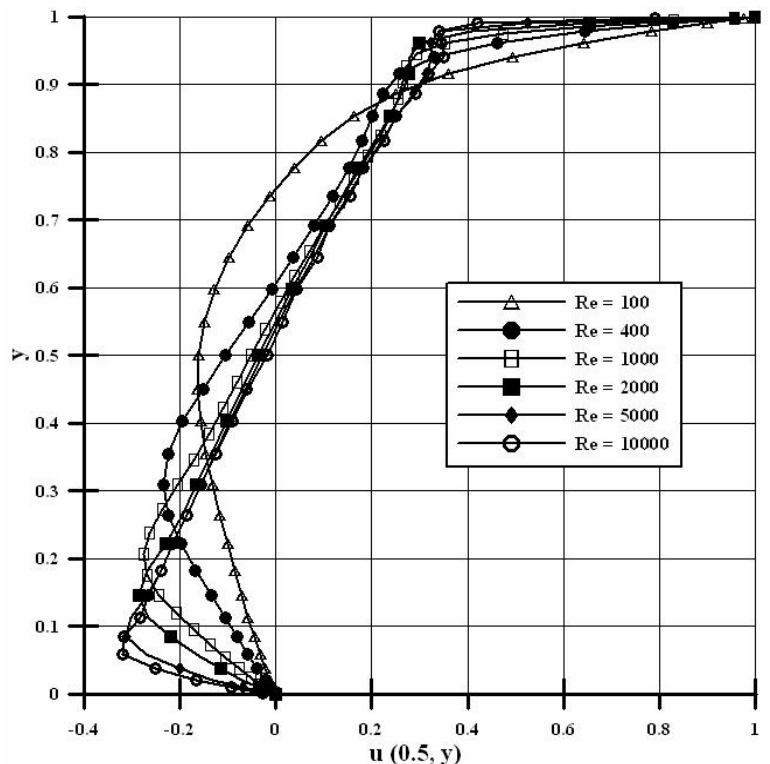

(a)

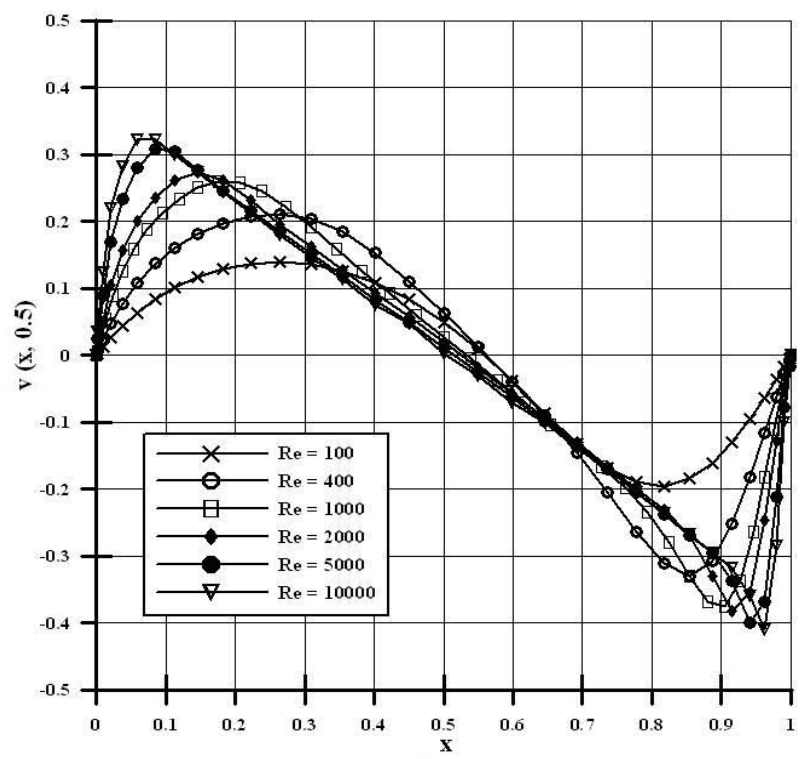

(b)

Figure 4. Variation of the velocity profiles on the centerline of the regularized driven cavity for some Reynolds numbers: (a) u -velocity, (b) v -velocity.

\section{Flow over a Backward-Facing Step}

The steady viscous incompressible flow over a backward-facing step is a benchmark problem that has been studied by numerous authors using a wide variety of numerical methods. Consider the area containing a step, as shown in Fig. 5. The channel is defined to have an unitary height $H$ with a step height localized in the upstream inlet region equal to $H / 2$ and the downstream channel length is $L=25 H$. The coordinate system to describe the locations in the channel is centered at the step corner. The definition of the problem as well as the nomenclature used follows Gartling (1990).

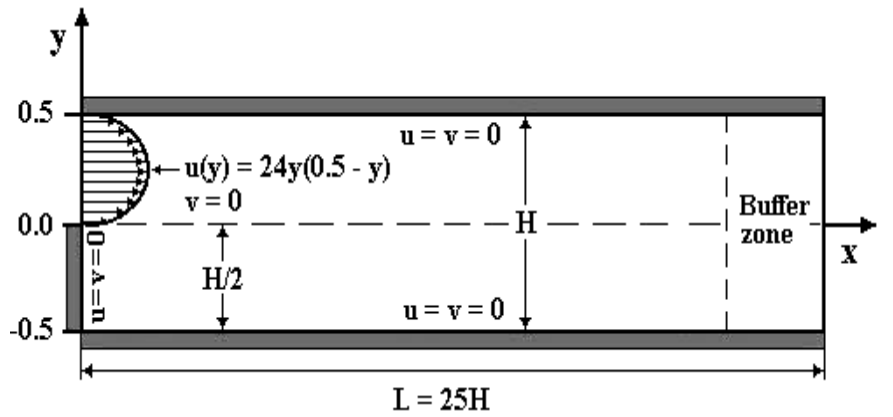

Figure 5. Geometry of the backward-facing step and boundary conditions.

The boundary conditions for the channel geometry are the noslip conditions for all walls. The inlet velocity field is specified as a parallel flow with a parabolic horizontal component defined by;

$$
u(y)=24 y(0.5-y) \quad \text { for } \quad 0 \leq y \leq 0.5
$$

This parabolic profile produces a maximum inflow velocity of $u_{\max }=1.5$ and an average inflow velocity $u_{\text {avg }}=1.0$. The outflow boundary condition used is a velocity field obtained from the parabolized Navier-Stokes incompressible equations and a Buffer zone is placed at the end of the channel (see Fig. 5). The Reynolds number is defined by the following relation:

$$
\operatorname{Re}=u_{\text {avg }} H / v
$$

A Buffer zone technique (Streett and Macaraeg, 1989/90) is implemented on a single domain. This technique recognizes the fact that the source of possible reflections from the outflow boundary is the elliptic nature of the Navier-Stokes equations arising from the viscous diffusion terms and from the pressure field. The idea is to remove this ellipticity at the outflow boundary. Then, the first source of ellipticity; the normal viscous terms are smoothly reduced to zero at the outflow boundary multiplying by a filter function $s_{j}$.

Similarly, the ability of the pressure field to carry signals back into the domain from the outer boundary is attenuated to zero at outflow by multiplying the source term of the pressure Poisson equation by the filter function. In the present simulations, the filter function utilized is a general function very similar to that used by Joslin et.al. (1991) which is expressed as

$$
s_{j}=\frac{1}{2}\left(1+\tanh \left\{4\left[1-2 \frac{\left(j-N_{b}\right)}{\left(N_{x}-N_{b}\right)}\right]\right\}\right)
$$

where $N_{b}$ is the number of the point that marks the beginning of the Buffer zone and $N_{x}$ is the number of the point that marks the position of the outflow boundary.

All numerical simulations for the backward-facing step flow were computed using a dimensionless channel length of $25 \mathrm{H}$, an appropriate grid of $91 \times 41$ points of Chebyshev collocations. The Buffer zone was set on point 79 of the grid (using 12 points of collocation in this zone) and the time step used in all simulations was 0.005 to guarantee the stability of the present method.

Table 4 shows the comparison of some characteristic values of the backward-facing step for $\mathrm{Re}=800$ with previously published numerical results obtained by Gartling (1990), that used the Galerkin finite element method with a grid of 8000 (400x20) elements with 9 nodes per element. Although we have used only a coarse grid of $91 \times 41$ collocation points of Chebyshev for all cases, 
the comparison of the positions of the separation and reattachments points shows good agreement.

Table 5 shows the comparison of lengths of recirculation regions in the backward-facing step for Reynolds number of 800 with numerical results obtained by others authors. The comparison of the present results shows good agreement with results of Kim and Moin (1985) and Gartling (1990). Kim and Moin (1985) used a finite difference method with a grid of $101 \times 101$.

Table 4. Comparison of some characteristic values of the backward-facing step for $\mathbf{R e}=\mathbf{8 0 0}$.

\begin{tabular}{|l|l|c|c|}
\hline Vortex & Localization (x, y) & $\begin{array}{c}\text { Gartling (1990) } \\
\text { Grid 400x40 }\end{array}$ & $\begin{array}{c}\text { Present Method } \\
\text { Grid 91x41 }\end{array}$ \\
\hline Top vortex & Separation point & $(4.85,0.50)$ & $(4.81,0.50)$ \\
& Reattachment point & $(10.48,0.50)$ & $(10.45,0.50)$ \\
\hline \multirow{2}{*}{ Bottom vortex } & Separation point & $(0.00,0.00)$ & $(0.00,0.00)$ \\
& Reattachment point & $(6.09,0.00)$ & $(6.00,0.00)$ \\
\hline
\end{tabular}

Table 5. Comparison of lengths of recirculation regions in the backward-facing step for $\mathbf{R e}=\mathbf{8 0 0}$.

\begin{tabular}{|l|c|c|c|}
\hline Lengths & $\begin{array}{c}\text { Kim and Moin (1985) } \\
\text { Grid 101x101 }\end{array}$ & $\begin{array}{c}\text { Gartling (1990) } \\
\text { Grid 400x40 }\end{array}$ & $\begin{array}{c}\text { Present Method } \\
\text { Grid 91x41 }\end{array}$ \\
\hline Upper wall separation zone & 5.75 & 5.63 & 5.64 \\
\hline Lower wall separation zone & 6.0 & 6.09 & 6.0 \\
\hline
\end{tabular}

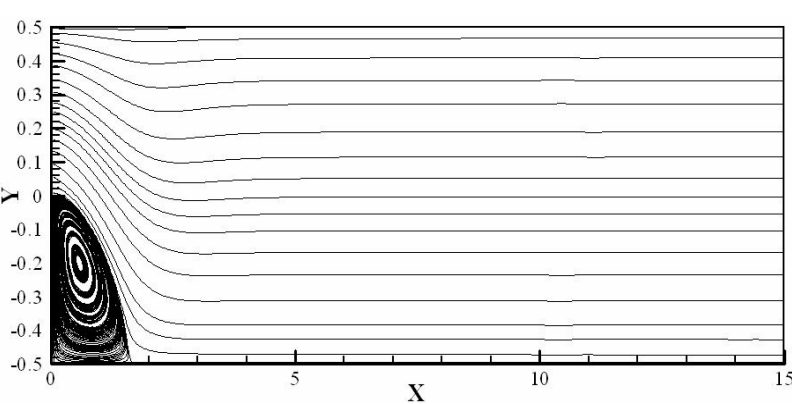

(a)

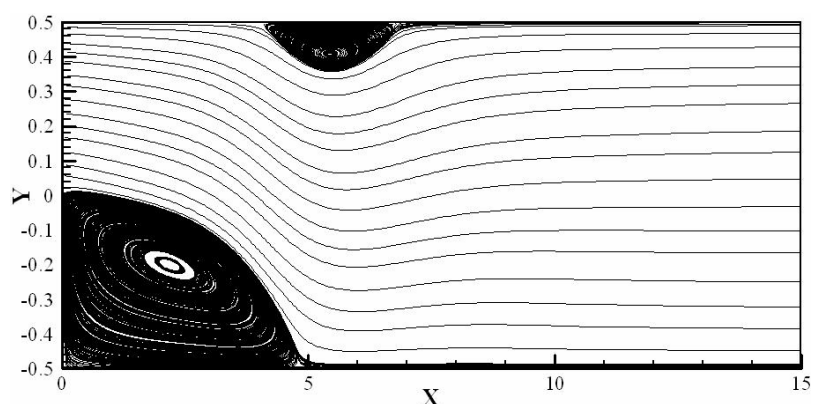

(b)

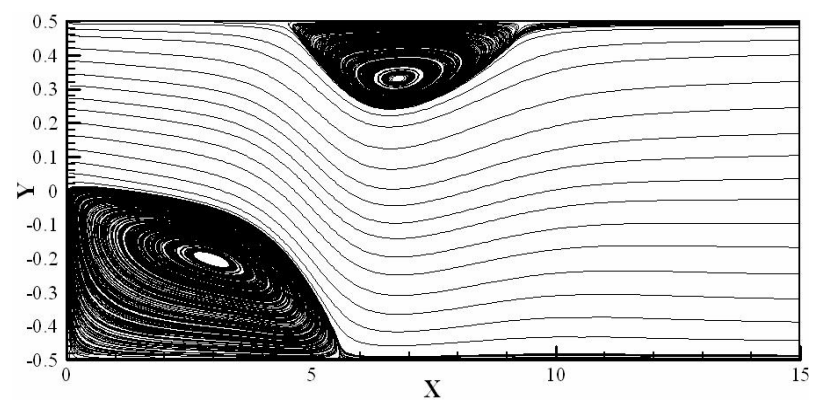

(c)

Figure 6. Steady state streamlines of the backward-facing step flow: (a) Re $=100$, (b) $\operatorname{Re}=500$, (c) $\operatorname{Re}=700$, (d) $\operatorname{Re}=800$, (e) $\operatorname{Re}=850$, (f) $\operatorname{Re}=875$ (Martinez, 2005).

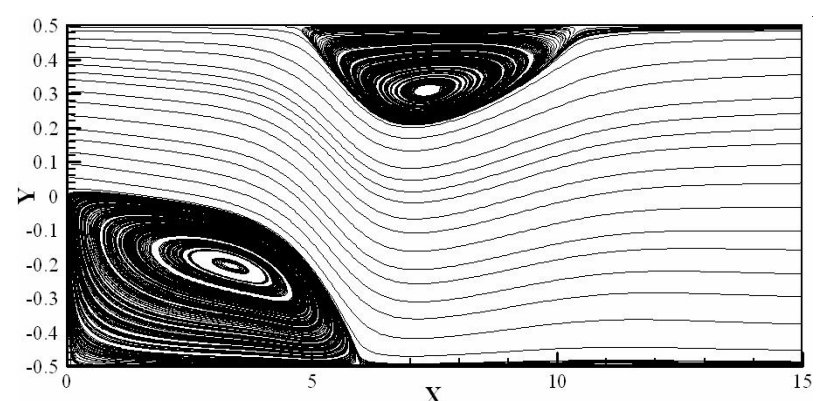

(d)

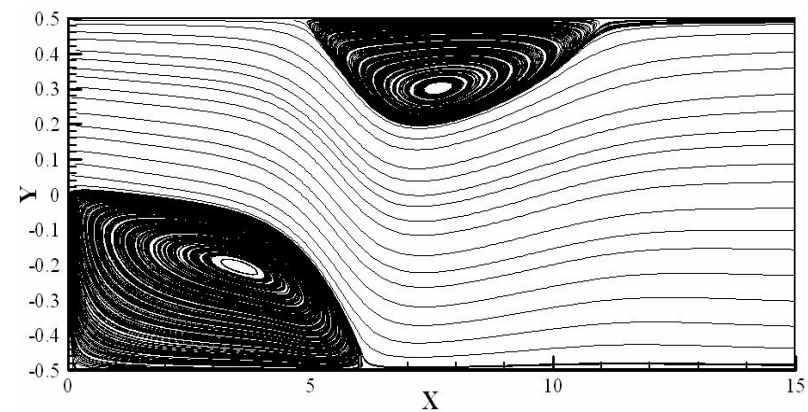

(e)

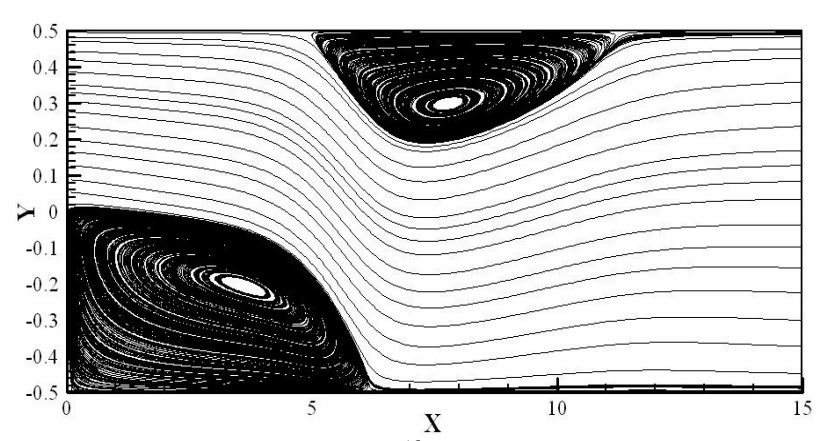

(f)

Figure 6. (Continued). 
Figures 6a - 6f show the steady state streamlines of the backward-facing step flow for Reynolds numbers up to 875 . Note that these figures only show the first 30 step heights $(H / 2)$ of the channel $(x=30(H / 2)=15)$.

In these figures, it can be observed the formation and growth of the vortices that appear at the top and bottom of the backwardfacing step when the Reynolds number increases. For example, for Reynolds numbers of 800 the flow separates at the step corner and forms a large recirculation eddy (Bottom vortex) with a reattachment point on the lower wall positioned approximately 12 step heights downstream $(x \approx 6)$. A second recirculation eddy (Top vortex) forms on the upper wall beginning approximately 10 step heights downstream $(x \approx 5)$ and finishing approximately at 21 step heights downstream $(x \approx 10.5)$. The figures show that the vortices for these Reynolds numbers ( $\mathrm{Re}=100,500,700,800,850$ and 875 ) can be very well represented using a coarse grid of $91 \times 41$ collocation points of Chebyshev.

Table 6 shows some characteristic values of the two separation zones that occur in the backward-facing step for Reynolds number of 875 .

Table 6. Some characteristic values of the separation zones of the backward-facing step for $\operatorname{Re}=\mathbf{8 7 5}$.

\begin{tabular}{|c|c|c|c|}
\hline & $\begin{array}{c}\text { Vortex centre } \\
(\mathbf{x}, \mathbf{y})\end{array}$ & $\begin{array}{c}\text { Separation } \\
\text { point }\end{array}$ & $\begin{array}{c}\text { Reattachment } \\
\text { point }\end{array}$ \\
\hline Top vortex & $(7.748,0.297)$ & $(4.97,0.50)$ & $(11.26,0.50)$ \\
\hline Bottom vortex & $(3.644,-0.206)$ & $(0.00,0.00)$ & $(6.14,0.00)$ \\
\hline
\end{tabular}

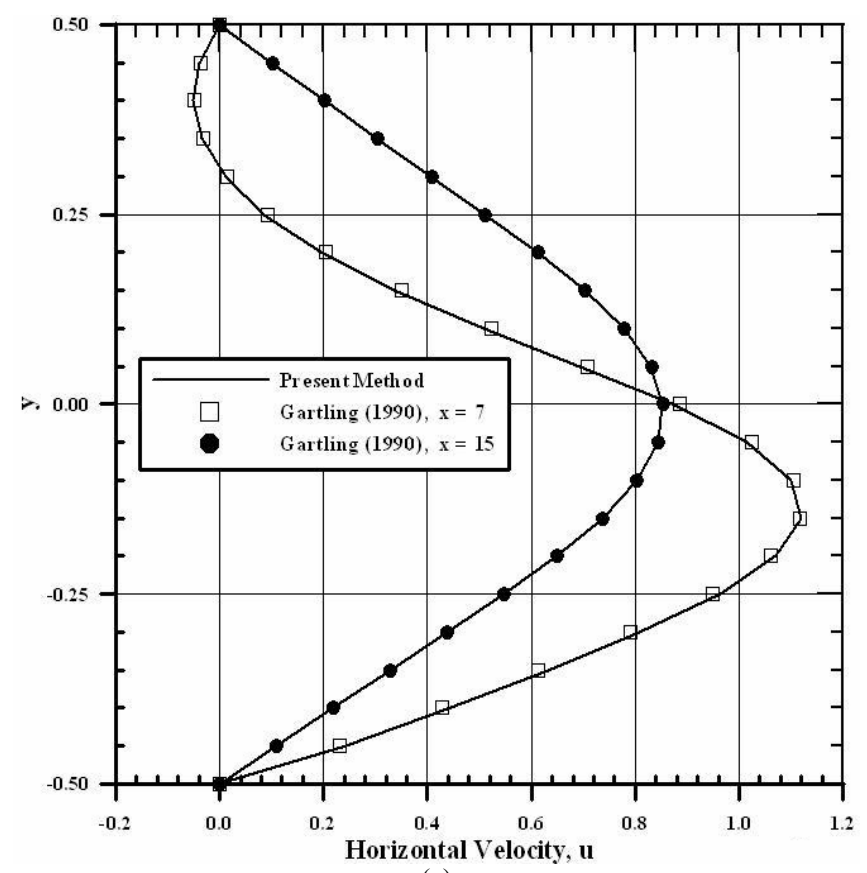

(a)

Figure 7. $u$-velocity component profiles across the channel at $x=7$ and $x=15:$ (a) $\operatorname{Re}=800$, (b) $\operatorname{Re}=875$.

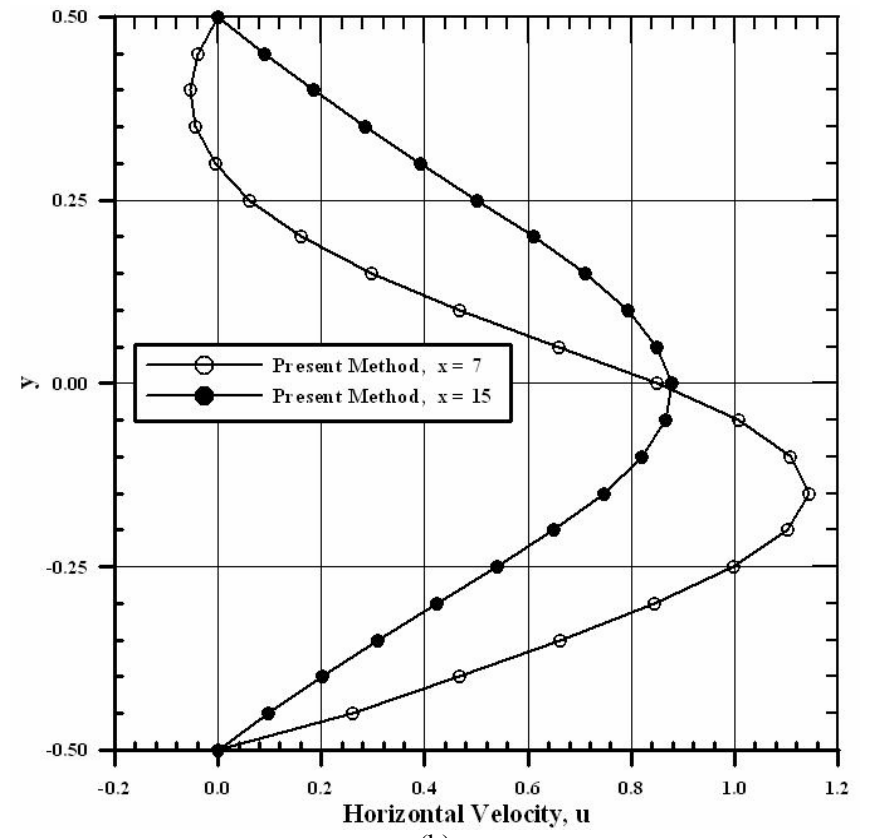

(b)

Figure 7. (Continued).

Figure 7 a shows the comparison of $u$ velocity component profiles across the channel located at $x=7$ (14 step heights downstream of the step) and $x=15$ (30 step heights downstream of the step) for Reynolds number of 800 with numerical results obtained by Gartling (1990). Here it can be observed the good agreement between velocity profiles obtained by the present method and those obtained by Gartling (1990). The variation of $u$ velocity component profiles across the channel located at $x=7$ and $x=15$ for Reynolds number of 875 is also shown in the Fig. 7b.

Finally, the comparison of vorticity profiles across the channel located at $x=7$ and $x=15$ for Reynolds number of 800 is shown in the Fig. 8a. Once again, can be noted the good agreement between vorticity profiles obtained by the present method and those obtained by Gartling (1990). Figure $8 b$ shows the variation of vorticity profiles across the channel located at $x=7$ and $x=15$ for Reynolds number of 875 .

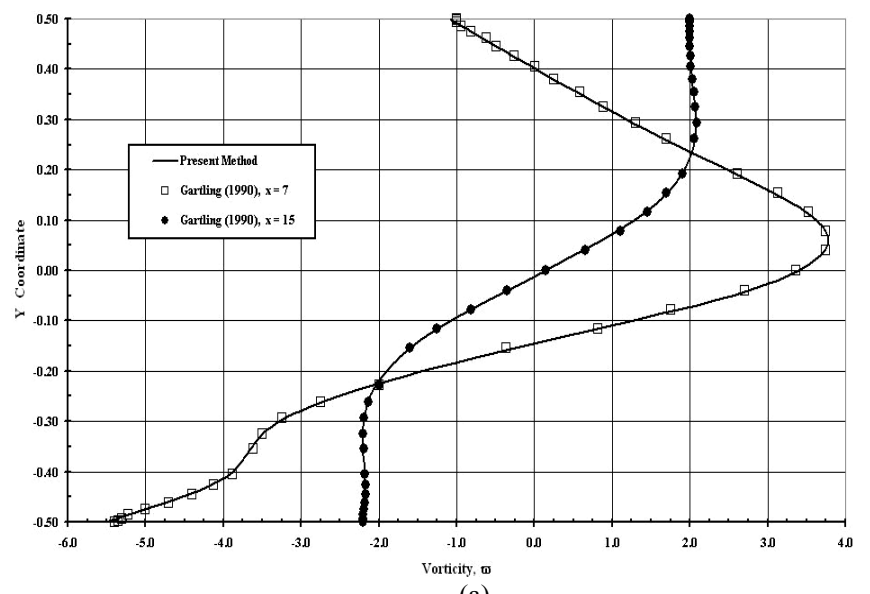

(a)

Figure 8. Vorticity profiles across the channel at $x=7$ and $x=15:$ (a) $\operatorname{Re}=800,(b) \operatorname{Re}=875$. 


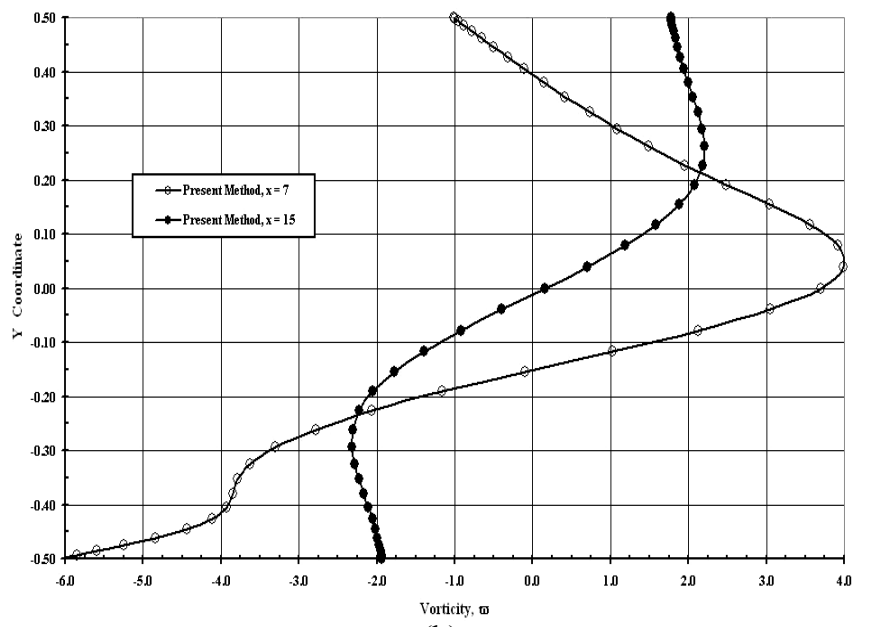

(b)

Figure 8. (Continued).

\section{Conclusions}

The projection method combined with the Chebyshev collocation spectral method associated with a second order explicitimplicit time scheme and appropriate boundary conditions, has shown to be a very stable scheme when applied to the solution of the Navier-Stokes equations for two-dimensional incompressible flow.

This combination of the projection scheme in conjunction with a Chebyshev collocation spectral method has been able to predict very well the behavior of the recirculating zones of the two-dimensional regularized square driven cavity flow for Reynolds numbers up to 10000 and the separation zones of the steady viscous incompressible flow over a backward-facing step for Reynolds numbers up to 875 . A good agreement was obtained from comparison of the numerical results obtained by the present method with available numerical solutions.

\section{Acknowledgements}

This work was sponsored by CAPES.

The calculations were performed on computer PC-Pentium III$700 \mathrm{MHz}$ of the Department of Ocean Engineering, Federal University of Rio de Janeiro, COPPE/UFRJ.

\section{References}

Botella, O., 1997, "On the Solution of the Navier-Stokes Equations using Chebyshev Projection Schemes with Third-Order Accuracy in Time", Computers \& Fluids, Vol. 26, No. 2, pp. 107-116.

Boyd, J.P., 2001, "Chebyshev and Fourier Spectral Methods", Ed. Springer-Verlag, Berlin, 688 p.

Brown, D.L., Cortez, R. and Minion, M.L., 2001, "Accurate Projection Methods for the Incompressible Navier-Stokes Equations", Journal of Computational Physics, Vol. 168, pp. 464-499.

Canuto, C., Hussaini, M., Quarteroni, A. and Zang, T.A., 2006, "Spectral Methods. Fundamentals in Single Domains", Ed. Springer-Verlag, Berlin, $585 \mathrm{p}$.
Chen, H., Su, Y. and Shizgal, B.D., 2000, "A Direct Spectral Collocation Poisson Solver in Polar and Cylindrical Coordinates", Journal of Computational Physics, Vol. 160, pp. 453-469.

Chorin, A.J., 1968, "Numerical Solution of the Navier-Stokes Equations", Mathematics of Computation, Vol. 22, pp. 745-762.

Deville, M., Fischer, P.F. and Mund, E.H., 2002, "High-Order Methods for Incompressible Fluid Flow", Cambridge Monographs on Applied and Computational Mathematics. Cambridge University Press, 499 p.

Ehrenstein, U. \& Peyret, R., 1989, "A Chebyshev Spectral Collocation Method for the Navier-Stokes Equations with Application to DoubleDiffusive Convection", International Journal for Numerical Methods in Fluids, Vol. 9, pp. 427-452.

Gartling, D.K., 1990, "A Test Problem for Outflow Boundary Conditions-Flow over a Backward-Facing Step", International Journal for Numerical Methods in Fluids, Vol. 11, pp. 953-967.

Ghia, U., Ghia, K.N. and Shin, C.T., 1982, "High-Re Solutions for Incompressible Flow using the Navier-Stokes Equations and a Multigrid Method", Journal of Computational Physics, Vol. 48, pp. 387-411.

Huges, S. \& Randriamampianina, A., 1998, “An Improved Projection Scheme Applied to Pseudospectral Methods for the Incompressible NavierStokes Equations", International Journal for Numerical Methods in Fluids, Vol. 28, pp. 501-521.

Joslin, R.D., Streett, C.L. and Chang, C.L., 1991, "Validation of 3D Incompressible Spatial Direct Numerical Simulation Code. A Comparison with Linear Stability and Parabolic Stability Equation Theories for Boundary Layer Transition on a Flat Plate", Report NASA Langley Research Center, NASA TP-3205, pp-1-47.

Kim, J. \& Moin, P., 1985, "Application of a Fractional-Step Method to Incompressible Navier-Stokes Equations", Journal of Computational Physics, Vol. 59, pp. 308-323.

Lynch, R.E., Rice, J.R. and Thomas, D.H., 1964, "Direct Solution of Partial Differential Equations by Tensor Product Matrices", Numerical Mathematics, Vol. 6, pp. 185-199.

Martinez, J.J., 1999, "Application of the Chebyshev Pseudospectral Method on some Hydrodynamic Problems" (In Portuguese), M.Sc. Thesis, Federal University of Rio de Janeiro, Rio de Janeiro, RJ, Brazil, 141 p.

Martinez, J.J., 2005, "Application of Spectral Multidomain Method for Simulation of Incompressible Viscous Flows" (In Portuguese), Ph.D. Thesis, Federal University of Rio de Janeiro, Rio de Janeiro, RJ, Brazil, 149 p.

Peyret, R., 2002, "Spectral Methods for Incompressible Viscous Flow", Applied Mathematical Sciences, Vol. 148, Ed. Springer-Verlag, New York, 448 p.

Shankar, P.N. \& Deshpande, M.D., 2000, "Fluid Mechanics in the Driven Cavity", Annual Review of Fluid Mechanics, Vol.32, pp. 93-136.

Shen, J., 1991, "Hopf Bifurcation of the Unsteady Regularized Driven Cavity Flow", Journal of Computational Physics, Vol. 95, pp. 228-245.

Streett, C.L. \& Macaraeg, M.G., 1989/90, "Spectral Multi-Domain for Large-Scale Fluid Dynamic Simulations", Applied Numerical Mathematics, Vol. 6, pp. 123-139.

Solomonoff, A. \& Turkel, E., 1989, "Global Properties of Pseudospectral Methods", Journal of Computational Physics, Vol. 81, pp. 239-276.

Tanahashi, T. \& Okanaga, H., 1990, “GSMAC Finite Element Method for Unsteady Incompressible Navier-Stokes Equations at High Reynolds Numbers", International Journal for Numerical Methods in Fluids, Vol.11, pp. 479-499.

Temam, R., 1968, "Une Méthode d'Approximation de la Solution des Équations de Navier-Stokes", Bulletin of Society Mathematics of France, Vol. 98, pp. 115-152.

Trefethen, L.N., 2000, "Spectral Methods in Matlab”, SIAM, University City Science Center, Philadelphia, USA, 165 p.

Zhao, S. \& Yedlin, M.J., 1994, “A New Iterative Chebyshev Spectral Method for Solving the Elliptic Equation", Journal of Computational Physics, Vol. 113, pp. 215-223. 